\title{
Reconstruction of Meibomian Gland Carcinoma - A Case Report
}

Dr. Kala Bagavathy, MDS, OMFS ${ }^{1 *}$, Dr.Priyesh Kesharwani, MDS $^{2}$, Dr. Rahul Vinay Chandra Tiwari, FOGS, MDS ${ }^{3}$, Dr. Jacob John Plackal ${ }^{4}$, Dr. Murali K ${ }^{5}$, Dr. Umesh Kaswan ${ }^{6}$

${ }^{1}$ Gokulam Hospital, Nagercoil, Tamil Nadu-62900 India

${ }^{2}$ Oral and Maxillofacial Surgeon, Consultant and Private Practitioner Dent-o-facial Multispeciality Clinic, Mira road, Thane-Mumbai India

${ }^{3}$ Assistant Professor Department of Oral and Maxillofacial Surgery, Sri Sai College of Dental Surgery, Vikarabad, India

${ }^{4}$ Consultant Oral and Maxillofacial surgeon, Believers Church Medical Centre, Konni, Pathanamthitta, Kerala India

${ }^{5}$ Consultant Plastic Surgeon, Chennai, Tamil Nadu India

${ }^{6}$ PG Student, OMFS, Surendra Dental College \& RI, Sriganganagar, Rajasthan, India

*Corresponding author: Dr. Kala Bagavathy, MDS, OMFS

\section{Abstract}

Meibomian gland carcinoma is an aggressive tumour with poor prognosis when diagnosed late. The tumour is often confused with neoplasms. This is a case report of a 40 year old male patient diagnosed with meibomian gland carcinoma. The tumour was resected completed along with orbital exenteration which was reconstructed with a FALT graft.

Keywords: Meibomian meibomian exenteration gland.

Copyright @ 2019: This is an open-access article distributed under the terms of the Creative Commons Attribution license which permits unrestricted use, distribution, and reproduction in any medium for non-commercial use (NonCommercial, or CC-BY-NC) provided the original author and source are credited.

\section{INTRODUCTION}

Meibomian gland carcinoma is a highly malignant, slow growing tumor of the eyelid. Terms such as sebaceous carcinoma, sebaceous gland carcinoma, and sebaceous cell carcinoma and meibomian gland carcinoma are used interchangeably to describe this neoplasm. It originates from the meibomian glands located in the tarsal plate, glands of Zeis, and sebaceous glands of caruncle and periocular skin [1]. With an incidence rate of $1-1.5 \%$, it accounts to nearly $3 \%$ of all the eye lid malignancies. This form of carcinoma is more prevalent amongst elderly females with a mean age of 57 to 72 years. This is more prevalent amongst the North American and Asian populations. Prior irradiation is an important risk factor. Numerous cases were reported in patients with familial retinoblastoma [2].

The neoplasm exhibits a multicentric pattern involving the other eyelid, conjunctiva or corneal epithelium, thus contributing to the lethal nature of the lesion. The metastatic growth spreads through the canaliculus to the lacrimal excretory system and even to the nasal cavity [3]. This carcinoma shows highly pleomorphic cells arranged in lobules or nests with hyperchromatic nuclei and a vacuolated cytoplasm. Histologically, the tumour should be differentiated from squamous cell carcinoma [4]. This is a rare case report of a 40year old male patient, diagnosed and treated for meibomian gland carcinoma.

\section{CASE REPORT}

A 40 year old man with a tobacco chewing habit reported to the ophthalmology OPD with a swelling in the right eye since 2 months. The patient was non-symptomatic 2 months back, after which he noticed a small, solitary swelling in his right eye, which increased rapidly within a month and attained the current size. Patient gives a history of recurrent blepharitis and conjunctivitis. Present day the nodularulcerative growth was measuring $3 \times 2.7 \mathrm{cms}$ in diameter, involving the upper eyelid, thus hindering the closure of the eye. On further examination, the patient presented with vertical dystopia along with restricted superior, superomedial and superolateral gaze. Diplopia was detected on superior gaze. Systemic examination revealed no regional or systemic lymphadenopathy [figure 1].

Microscopic examination of an incisional biopsy revealed stratified squamous epithelium. The underlying tumour cells were composed of sheets and lobules of cells showing large, hyperchromatic nuclei and prominent nucleoli and abundant cytoplasm. Few mitotic figures were noted along with a spindle shaped stroma, suggestive of meibomian gland carcinoma. Magnetic resonance imaging of the orbits showed a lobulated mass in the superiolateral aspect of right orbit involving the superior rectus muscle. The mass involved only the preseptal compartment with no evidence of intracranial extension. The roof of the orbit 
and optic nerve showed no signs of tumour involvement.

Orbital exenteration involving the roof of orbit along with superficial parotidectomy was done, to completely resect the tumour along with $5 \mathrm{~mm}$ of healthy tissue. Frontal sinus mucosa, inferior ophthalmic nerve, optic nerve was sent for frozen section (FS). They were negative for malignancy. A Modified blair incision was given and digastric was dissected. Two lymph nodes from level II were dissected and sent for FS which showed reactive nodes. Tragal cartilage was exposed and dissected lower down till the junction with digastric muscle. Posterior branch of greater auricular nerve was preserved. Facial nerve was identified. Superficial parotidectomy along with cervical branch of facial nerve was cut during dissection. All other branches were preserved in continuity. Hemostasis was achieved. The defect was reconstructed with FALT (free flap anterolateral thigh) graft [figure 2, figure 3, and figure 4].

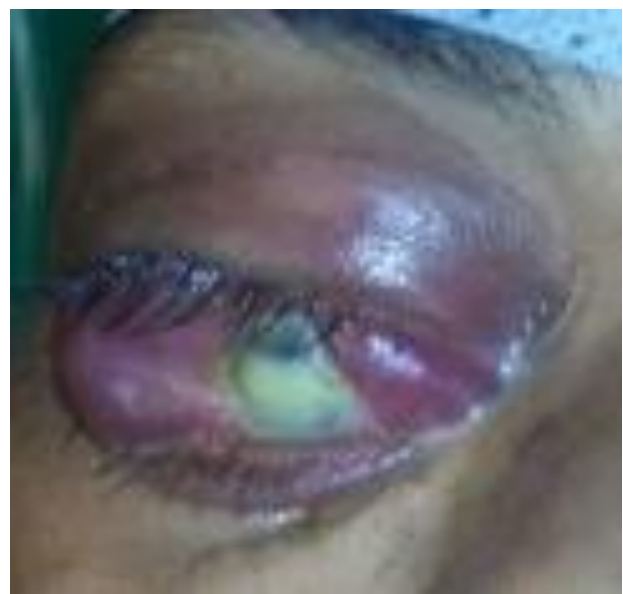

Fig-1: Pre-operative

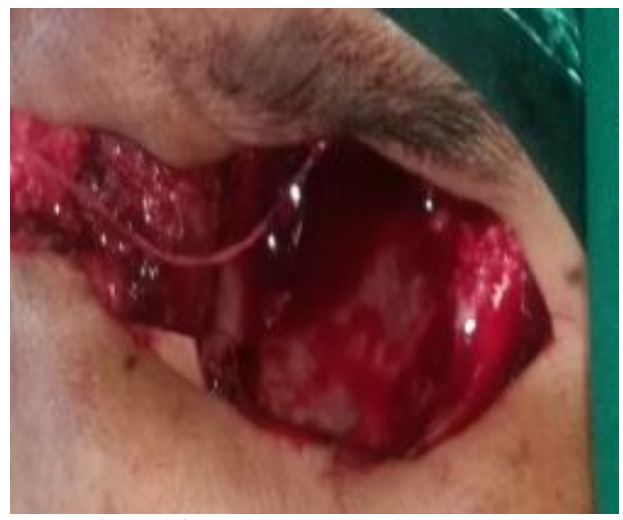

Fig-2: After complete surgical resection

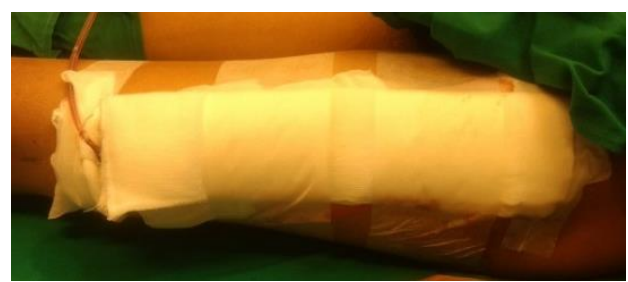

Fig-3: Graft site

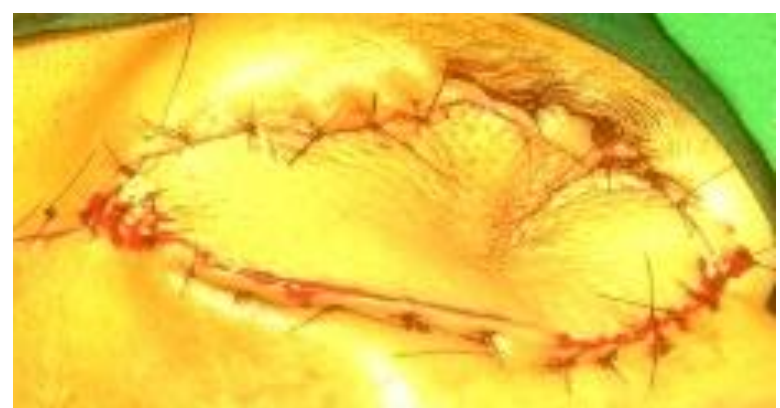

Fig-4: Immediate postoperative after reconstruction of the orbit

\section{DISCUSSION}

Meibomian gland carcinoma is a rare, rapidly progressive tumor arising from the meibomian gland in the tarsus and glands of Zeis present in the eyelashes. It is the third most common malignancy in the eyelid. Studies reported an increased incidence of sebaceous gland carcinoma in the Asian population [5]. This tumour presents in two forms, peri-ocular and extraocular, peri-ocular being the most common type. Due to the presence of increased number of meibomian glands in the upper eyelid, the lesion more commonly originates in the upper eyelid than lower eyelid. Kass and Hornblass reported an incidence rate of $63 \%$ in the upper eyelid and $27 \%$ in the lower eyelid and $5 \%$ involving both eyelids [6]. Rao et al. reported in their review that $10 \%$ of the cases arise from the Zeis glands and only $5-10 \%$ of the lesions originate from the Zeis glands [7]. The advanced malignancies are usually multicentric in origin, which spread through the pagetoid, which constitute $18 \%$ of the advanced tumours. Extraocular sebaceous carcinoma is seen in the head and neck area. In our case report, carcinoma was a peri-ocular type involving the upper eyelid.

The diagnosis of meibomian gland carcinoma is often mistaken for blepharoconjunctivitis or a chalazion, thus delaying the exact diagnosis. The delay increases the chance of local recurrence, metastasis and death. The physicians should be aware of different variants of this carcinoma. The usual variants are solitary eyelid nodule, diffuse pseudo-inflammatory pattern, and pedunculated lesion, eyebrow mass and lacrimal gland mass. Solitary eyelid nodule is the most common clinical variant, which develops as a painless, firm, sessile to round, subcutaneous nodule in the eyelid. The skin over the lesion is generally smooth and fairly movable. As the tumour enlarges and encroaches on the epidermis, it assumes a yellow colour due to the presence of lipid in the mass. When the tumour arises from the Zeis gland, it can appear as a rounded mass at the eyelid margin, without such a firm attachment to the tarsus [8].

It should be differentiated from conjunctivitis, keratoconjuctivitis, superior limbic keratoconjunctivitis, and tumours like basal cell carcinoma, squamous cell carcinoma, melanoma, merkel cell carcinoma and 
lymphoma. Recurrent chalazion or unilateral blepharoconjunctivitis in elderly individuals along with loss of lashes should be biopsied.

Based on the histopathological features, sebaceous carcinoma can be differentiated into lobular, comedocarcinoma, papillary and mixed carcinoma variant. Lobular pattern is the most frequent histopathological pattern detected with less differentiated cells situated peripherally, and well differentiated, lipid producing cells located centrally. In comedocarcinoma variant, the lobules show a necrotic central core surrounded by peripheral viable cells. The papillary pattern occurs frequently in small conjunctival tumors characterized by papillary projections and areas of sebaceous differentiation. The characteristic feature of this carcinoma is the pagetoid pattern of invasion, in which the tumour spreads into eyelid epidermis and conjunctival epithelium. In the case we reported, histopathologically, biopsy section presented with a lobular pattern of cells [9].

Investigations such as an incisional biopsy are done to establish the diagnosis. In case of extensive and diffuse invasion, orbital imaging studies are done to delineate the extension of the tumour. Fine needle aspiration cytology (FNAC) and impression cytology have limited application in diagnosis of sebaceous gland carcinoma.

Establishing an accurate diagnosis and extent of the disease accurately is of paramount importance in determining the management of the tumour. A comprehensive clinical evaluation of the eyelid, conjunctiva, caruncle and adjacent areas should be done. Palpation of preauricular and cervical areas should be performed to detect possible lymph node metastasis. The following goals should be followed to establish a treatment plan:

- Control the extent of the tumour

- Restore the eye globe and vision.

- Restore the cosmetic appearance

Usually a combination of approaches will be adopted depending on the overall clinical circumstances. Excisional biopsy which involves complete surgical excision of the lesion is usually preferred if the lesion is small and circumscribed. For meibomian gland carcinoma, a full-thickness, pentagonal, eyelid resection along with $5 \mathrm{~mm}$ of nasal and temporal margins is preferred. A lateral semicircular flap is reflected to achieve primary closure of the wound. Incisional biopsy is indicated in diffuse blepharoconjunctivitis and in advanced primary lesions requiring reconstruction after excision. At the time of excision, either frozen section or Mohs microsurgery is often used to check the margins for residual tumour until the margins are clear histopathologically[10, 11].
Treatment of sebaceous gland carcinoma is primarily surgical. Surgical treatment may range from a local excision to orbital exenteration. Radical surgical excision with frozen section control by a standard method or Mohs micrographic surgery is the most common and effective method of treatment. Excision of $4-5 \mathrm{~mm}$ of normal tissue along with the tumour cells improves the overall prognosis. Distant metastasis requires adjuvant chemotherapy and radiotherapy. Involvement of upper or both eyelids, tumor size of $\geq 10$ $\mathrm{mm}$, poorly differentiated tumours, infiltration into blood vessels and lymphatics, orbital extension, multicentric origin, and pagetoid pattern of invasion are the poor prognostic factors for meibomian gland carcinoma [12].

The reconstruction of the exenteration defects with flaps can be considered to close the defects. The commonly used flaps are: rectus abdominis musculocutaneous flap, forearm-based flaps and antero-lateral thigh flap (FALT). Amongst these, FALT is the most preferred graft in head and neck reconstruction. Advantage of this graft is that it can be harvested both as a purely fascio-cutaneous flap or musculo cutaneous flap to obliterate dead space. Other advantages are its long pedicle and low donor site morbidity, which could either, is closed primarily or covered with a split thickness skin graft [13].

\section{CONCLUSION}

Meibomian gland carcinoma is often misdiagnosed as inflammatory disease or other tumours, resulting in delay in diagnosis, hence increasing the morbidity and mortality rate. Increased awareness has resulted in earlier diagnosis and has provided the opportunity for less aggressive therapy. Today, more cases are being managed by carefully planned map biopsies, local resection, and advanced methods of reconstruction, combined with cryotherapy and topical chemotherapy. With increased awareness, improved medical access and newer treatment options, orbital exenteration can be avoided to improve the patient survival rate.

\section{REFERNCES}

1. Shields, J. A., Demirci, H., Marr, B. P., Eagle Jr, R. C., \& Shields, C. L. (2005). Sebaceous carcinoma of the ocular region: a review. Survey of ophthalmology, 50(2), 103-122.

2. Marianela, C. F., Hector, P. R. L., \& Pacheco, A. B. C. (2018). Sebaceous Cell Carcinoma of the Lower Eyelid-A Case Report. Journal of Clinical Research in Oncology, 1(1), 1-3.

3. Huang, S. Y., Chen, Y. W., Liao, Y. H., Wu, C. T., Wang, T. H., Hsu, W. L., \& Liu, D. W. (2018). Sebaceous carcinoma of eyelid treated with definitive radiotherapy with eye shield. Therapeutic Radiology and Oncology, 2.

4. Mohta, A., Sabnis, M., \& Nayyar, M. (2018). A Rare Case of Eye Lid Malignancy: Meibomian 
Gland Carcinoma. Journal of Advanced Medical and Dental Sciences Research, 6(6), 24-26.

5. Maurya, R. P., Singh, V. P., Singh, M. K., Jain, P., \& Kuwanr, A. (2015). Recurrent Meibomian gland carcinoma of lower eyelid in male: A case report. Ind J ClinExpOphthalmol, 1(3), 181-183.

6. Kass, L.G., Hornblass, A.M. (1989). Sebaceous carcinoma of the ocular adnexa. Surv Ophthalmol, 33:477-90.

7. Rao, N. A., Hidayat, L. C. A. A., McLean, L. C. I. W., \& Zimmerman, L. E. (1982). Sebaceous carcinomas of the ocular adnexa: a clinicopathologic study of 104 cases, with five-year follow-up data. Human pathology, 13(2), 113-122.

8. Shields, J.A., Demirci, H., Marr, B.P., Eagle, Jr R.C., Shields, C.L.(2005). Sebaceous carcinoma of the ocular region: a review. Survey of ophthalmology, 1;50(2):103-22.

9. Dasgupta, T., Wilson, L.D., Yu, J.B.(2009). A retrospective review of 1349 cases of sebaceous carcinoma. Cancer, 115(1):158-65.
10. Bailet, J.W., Zimmerman, M.C., Arnstein, D.P., Wollman, J.S, Mickel, R.A.(1992). Sebaceous carcinoma of the head and neck: case report and literature review. Archives of OtolaryngologyHead \& Neck Surgery, 118(11):1245-9.

11. Mulay, K., Aggarwal, E., White, V.A. (2013). Periocular sebaceous gland carcinoma: a comprehensive review. Saudi Journal of Ophthalmology, 27(3):159-65.

12. Lam, S.C., Li E.Y., Yuen H.K. (2018). 14-year case series of eyelid sebaceous gland carcinoma in Chinese patients and review of management. British Journal of Ophthalmology, 102(12):1723-7.

13. Ogawa, M., Shinzawa, M., Dogru, M., Miyauchi, J., Tanaka, Y., Ogawa, Y., Obata, H., Tsubota, K., Shimazaki, J. (2018). Caruncular and pericaruncular sebaceous gland hyperplasia: a report of 2 cases and literature review. Eye \& contact lens, 1 ; 44: S316-9. 